\title{
3 Research Square

\section{Metabolic-Associated Signature and Hub Genes Associated with Immune Microenvironment and Prognosis in Bladder Cancer}

\section{Yadong Guo}

Shanghai Tenth People's Hospital

\section{Zongtai Zheng}

Shanghai Tenth People's Hospital

\section{Shiyu Mao}

Shanghai Tenth People's Hospital

\section{Fuhan Yang}

Shanghai Tenth People's Hospital

\section{Ruiliang Wang}

Shanghai Tenth People's Hospital

\section{Hong Wang}

Shanghai Tenth People's Hospital

Ji Liu

Shanghai Tenth People's Hospital

Cheng Li

Shanghai Tenth People's Hospital

Wentao Zhang

Shanghai Tenth People's Hospital

Shenghua Liu

Shanghai Tenth People's Hospital

Xu-dong Yao ( $\nabla$ yaoxudong1967@163.com )

Shanghai Tenth People's Hospital https://orcid.org/0000-0001-7234-3940

\section{Research}

Keywords: bladder cancer, metabolism, immune microenvironment, prognosis, risk signature

Posted Date: November 11th, 2021

DOI: https://doi.org/10.21203/rs.3.rs-1041335/v1 
License: (c) (i) This work is licensed under a Creative Commons Attribution 4.0 International License. Read Full License 


\section{Abstract}

Background: The relationship between metabolism and immune microenvironment remains to be studied in bladder cancer (BCa). We aimed to construct a metabolic-associated signature for prognostic prediction and investigate its relationship with the immune microenvironment in $\mathrm{BCa}$.

Methods: The RNA expression of metabolism associated genes was obtained from a combined data set including The Cancer Genome Atlas, GSE48075, and GSE13507 to divide BCa patients into different clusters. A metabolic-associated signature was constructed using the differentially expressed genes between clusters in the combined TCGA and validated in the IMvigor210 trial and our center. The sensitivity of metabolic-associated signature to chemotherapy and immunotherapy for predicting $\mathrm{BCa}$ was assessed. The proportion of 28 subtypes of tumor-infiltrating immune cells (TIICs) in each BCa sample was assessed using a single sample gene set enrichment analysis (ssGSEA). TST and S100A16 were identified by hub genes screening. Survival analysis and nomograms were used to evaluate the prognosis and predictive value in BCa based on TST and S100A16. The loss of function study further investigated the effects of TST and S100A16 on the biological function and immune microenvironment of $\mathrm{BCa}$ cells.

Results: BCa patients in high-risk group were associated with advanced clinicopathological features and poor survival outcomes. The percentage of high-risk patients was significantly lower in patients responding to anti-PD-L1 treatment (complete response/partial response). Compared with low-risk patients, the IC50 values of cisplatin and gemcitabine were significantly lower in high-risk patients. sSGSEA revealed that the composition of TIICs including macrophages and regulatory $T$ cells between risk groups. TST and S100A16 were significantly associated with clinicopathological features and prognosis. Down-regulation of TST promoted BCa cell invasion, migration, and EMT, which are inhibited by down-regulation of S100A16. CD8+T cells, neutrophils, and dendritic cells had higher infiltration in the TST low-group and the S100A16 high-group. Furthermore, loss of function TST and S100A16 significantly affected the expression of PD-L1 and CD47.

Conclusion: The metabolic-associated signature can stratify BCa patients into distinct risk groups with different immunotherapeutic susceptibility and survival outcomes, may contribute to effective individualized treatment. Dysexpression of TST or S100A16 contributes to the tumor progression and dysregulation of immune microenvironment in $\mathrm{BCa}$.

\section{Introduction}

Bladder cancer $(\mathrm{BCa})$ ranks the 10th most common malignancy in the world, with approximately 17,980 deaths and 81,400 new cases in the USA in 2020 (1). BCa can be classified into muscle-invasive bladder cancer (MIBC, 25\%) and non-muscle-invasive bladder cancer (NMIBC, 75\%) (2). NMIBC patients typically receive the transurethral resection of bladder tumor (TURBT) followed by intravesical therapy with Bacillus CalmetteGuérin (BCG) for patients with carcinoma in situ or high-grade T1 (3). As for MIBC 
patients, radical cystectomy or chemotherapy is typically recommended $(4,5)$. To date, the treatment options for BCa patients are still limited, and the rates of recurrence, progression and metastasis are still disappointingly high $(6,7)$. In addition, the treatment strategy and prognosis prediction mainly rely on tumor staging system, which is not sufficient to accurately predict the prognosis and develop the personalized treatment in $\mathrm{BCa}$ (8). Therefore, exploration of effective biomarkers for prognosis prediction and clinical management is still challenging.

Dysregulations of the metabolism and immune microenvironment within tumor are key events in various tumors $(9,10)$. Previous studies have reported that the dysregulation of some metabolic pathways, including activation of fatty acid metabolism, glutamine metabolism and glycolysis, are highly related to the tumorigenesis and tumor progression in BCa $(11,12)$. In addition, studies have predicted the prognosis of $\mathrm{BCa}$ patients and treatment response by metabolic classification, which indicates that metabolism plays an important role in $\mathrm{BCa}$ (13). Thus, exploration of metabolism associated genes (MAGs) may be useful in discovering the key metabolic-associated mechanisms controlling carcinogenesis, which may facilitate the development of new treatment options. Furthermore, some studies have found important effects of metabolism on immune cells in tumors, which lead to the tumor microenvironment being generally acidic, hypoxia and/or depletion of key nutrients needed by immune cells (14). It has been reported that tumor metabolites affect the function of tumor-associated macrophages (TAMs), and TAMs further activate glycolysis, fatty acid synthesis, and alter nitrogen cycle metabolism by reprogramming their metabolism to promote tumor progression and metastasis (15). Moreover, macrophage subtypes associated with immunosuppression, tumor angiogenesis, and progression affect the progression of $\mathrm{BCa}(16)$. However, no systematic assessment of the association between metabolism phenotype and immune microenvironment (including tumor-infiltrating immune cells (TIICs) and the expression of critical immune checkpoint genes) has been performed in BCa.

In this study, we used the transcriptomic data to establish and validate a metabolic-associated signature for prognostic prediction. In addition, we further investigated the relationship between the metabolicassociated signature/hub genes and the immune microenvironment.

\section{Results}

\section{Consensus Clustering Identified Two Clusters of BCa patients}

The expression of 1257 MAGs was achieved from TCGA, GSE48075 and GSE13507. These three data sets (712 patients) were combined for clustering. We divided the combined data set into several clusters based on the consensus of expression of 1257 MAGs (Figure 1).

When the ' $\mathrm{k}$ ' (clustering index) increased from 2 to $9, \mathrm{k}=2$ was the optimal value to obtain the minimal interference (Figure 2A) and largest differences (Figure 2B) between clusters. In this way, the combined data set was classified into two robust clusters, namely Cluster A and Cluster B. Kaplan-Meier survival analysis revealed that Cluster A had a significantly poor OS than Cluster B (Figure 2C). Cluster A was significantly associated with advanced clinicopathological features, including tumor stage, tumor grade, 
histological subtype and age (Figure 2D). GSVA demonstrated that infection-related biological processes and metabolic-related biological processes were mainly enriched in Cluster $A$ and Cluster $B$, respectively (Figure 2E). ssGSEA showed that the proportions of 27 TIICs were different between Cluster A and B (Figure 2F).

\section{Development of the metabolic-associated signature}

There were 1721 DEGs between Cluster A and B. After the feature filtering by the random forest and the univariate cox regression, 159 genes ( $H R>1$ : 56 genes, HR<1: 103 genes; SupplementaryTable2) were selected for the development of the metabolic-associated signature using the formula mentioned above. In the combined data set, BCa patients were divided into low- and high-risk groups based on the optimal cutoff value (-0.03655953). Patients in the high-risk group had significantly poor survival outcomes than patients in the low-risk group (Figure 3A). In addition, the IC50 values of Cisplatin and Gemcitabine were significantly higher in patients with low-risk scores (Figure 3B,C).

In TCGA, the proportions of 26 TIICs were different between low- and high-risk groups (Figure 3D), and patients with malignant clinicopathological features, including non-papillary subtype, high tumor grade, high tumor stage, older in age ( $\geq 60$ years), female and smoking history, had significantly higher risk scores (Figure 3E). In addition, patients with high-risk scores exhibited poor OS compared with those with low-risk scores (Figure 3F).

\section{Genetic Alteration in CCLE}

592 carcinoma cell lines were divided into low- and high-risk groups based on the median cutoff value $(-0.06701084)$. The top 20 mutation genes in low- and high-risk groups were presented in Supplementary Figure 1, which revealed the difference in genetic alteration between low- and high-risk groups. In addition, carcinoma cell lines with low-risk scores had significantly higher mutation than those with highrisk scores (Figure 3G).

\section{Validation of the metabolic-associated signature}

In IMvigor210 trial, patients treated with PD-L1 inhibitor were divided into low- and high-risk groups based on the optimal cutoff value (0.6055458). Kaplan-Meier survival analysis revealed that high-risk patients had significantly poor OS than low-risk patients (Figure 4A). In addition, the percentage of high-risk patients was significantly lower in patients responding to anti-PD-L1 treatment (complete response $(\mathrm{CR}) /$ partial response (PR)) than in patients non-responding to anti-PD-L1 treatment (progressive disease (PD)/stable disease (SD)) group (Figure 4B), which indicating the potential clinical value of the metabolic-associated signature to identify the immunotherapeutic susceptibility for patients with BCa.

In our center a total of $135 \mathrm{BCa}$ samples [108 male, 27 female; mean age $=70.4$ years \pm 11.7 (standard deviation), range 31-95 years; 99 NMIBC, 36 MIBC; 30 low-grade, 105 high-grade] were collected for RNAsequence. High-grade patients and MIBC patients had significantly higher risk scores than NMIBC patients and low-grade patients, respectively $(P<0.001$ and $P=0.003$, Figure 4C,D). 


\section{The prognostic value of hub genes}

Through hub genes screening, three genes, including Thiosulfate sulfurtransferase (TST), S100 calcium binding protein A16 (S100A16) and Matrix metalloproteinase 9 (MMP9) were obtained (Figure 5A). The functions of MMP9 have been fully investigated in various tumors (17), so the experimental validation of TST and S100A16 was performed in this study. Validation in the Human Protein Atlas shows that the protein levels of TST and S100A16 in BCa are different from those in normal bladder tissues, especially S100A16 (Figure 5B,C). In addition, RNA-sequence data in our center showed that the expressions of TST and S100A16 were significantly correlated with T stage (Figure 5D) and histopathological grade (Figure 4E). The Assistant for Clinical Bioinformatics and Kaplan Meier plotter database analysis showed that the low expression group of TST and the high expression group of S100A16 predicted poor diseasespecific survival (DSS) and OS of BCa patients (all $P<0.05$, Figure $5 F, G$ ).

Univariate Cox proportional hazards regression analysis showed that age, pTNM stage, low expression of TST and high expression of S100A16 were associated with poor OS of BCa patients in TCGA (Figure 6A). Then, multivariate Cox regression analysis showed that age, pTNM stage, TST and S100A16 were independent prognostic factors of BCa patients (Figure 6B). Then, we combined age, TST and S100A16 to generate a prognosis nomogram. The results showed that the nomogram could usefully predict the 1year, 3-year and 5-year survival outcomes of BCa patients (Figure 6C,D). Taken together, these findings indicate that TST and S100A16 are significantly related to the clinicopathological features and prognosis of BCa patients, which indicates the importance of further research.

\section{Downregulation of TST promoted the invasion, migration and EMT of BCa cells, while down-regulation of S100A16 inhibited those processes}

We synthesized two specific sgRNAs for TST and S100A16 respectively, and studied whether TST and S100A16 affected the migration and invasion of BCa using matrigel drop infiltration assay and transwell migration assay. Firstly, we confirmed by western blot that the expression of TST and S100A16 in T24 cells can be effectively knocked out by sgRNA (Figure 7A). Then matrigel droplet measurement was performed, and the radial distance of cells moving out from the edge of matrix glue droplet was measured on the 4th day after spreading the cells. Compared with the control group, the down-regulation of TST enhanced the invasion ability of BCa cells, while down-regulation of S100A16 inhibited these processes (Figure 7B-E). Similarly, we measured the migration of T24 cell by transwell migration assay, which was consistent with the above results. The results showed that downregulation of TST enhanced the invasion and migration of BCa cells, while down-regulation of S100A16 inhibited those processes (Figure 7F-I).

Next, in order to explore the detailed mechanism of TST and S100A16, we used GSEA to analyze the TST and S100A16 related signal pathways of BCa in TCGA database. GSEA analysis showed that TST-low group and S100A16-high group were significantly positive in FOCAL ADHESION, GAP JUNCTION (Figure $7 \mathrm{~J}, \mathrm{~K}$ ) and ADHERENS JUNCTION, which means that TST and S100A16 may play a key role in the progression of tumor epithelial-to-mesenchymal transition (EMT). Based on this, the protein expression 
level of EMT markers were detected. These results showed that E-cadherin decreased, while mesenchymal markers including $\mathrm{N}$-cadherin, vimentin and slug increased in T24 cell line in which knocked out TST (Figure 6L). Conversely, E-cadherin was upregulated and $\mathrm{N}$-cadherin, vimentin and slug were downregulated in T24 cell line which knocked out S100A16 (Figure 6M). These results indicate that TST and S100A16 play a vital role in invasion, migration and EMT of BCa cells.

\section{Correlation between TST and S100A16 and tumor immune microenvironment of BCa}

GSEA analysis showed that the gene sets of BLADDER CANCER, ANTIGEN PROCESSING AND PRESENTATION, NATURAL KILLER CELL MEDIATED CYTOTOXICITY, CYTOSOLIC DNA SENSING PATHWAY were significantly enriched in patients with lower levels of TST expression and higher levels of S100A16 (Figure 8A,B; Supplementary Figure 2A,B). The results of the Assistant for Clinical Bioinformatics showed that $C D 8^{+} T$ cells, neutrophils and dendritic cells had higher immune infiltration in the TST-low group and S100A16-high group (Supplementary Figure 2C,D).

And then, the correlation between TST, S100A16 and immune cell infiltration and the influence of different immune cells on the prognosis of BCa were analyzed by using TIMER database. According to the results of TIMER, the expression of TST was negatively correlated with the infiltration of $\mathrm{CD} 8^{+} \mathrm{T}$ cells, $\mathrm{CD}^{+} \mathrm{T}$ cells, neutrophils and dendritic cells, while the expression of S100A16 was positively correlated with these immune cells $(P<0.05$, Supplementary Figure $2 E, F)$. TIMER for 'survival' module showed that the high expression of $\mathrm{CD}^{+} \mathrm{T}$ cells and S100A16 had worse prognosis, while patients with high expression of TST had better prognosis (all $\mathrm{P}<0.05$, Supplementary Figure $2 \mathrm{G}$ ).

Furthermore, we analyzed the correlation between TST and S100A16 in BCa and immune-related checkpoints in TCGA. Our results showed that TST and S100A16 were significantly correlated with most immune-related checkpoints, especially PD-L1 and CD47 ( $<<0.05$, Supplementary Figure $2 \mathrm{H}, \mathrm{I})$. To test whether TST and S100A16 really participate in the regulation of PDL1 and CD47, we transfected T24 cell with TST and S100A16 specific Cas9/sgRNA, and detected the expression of PD-L1 and CD47 at mRNA and protein levels. Our data showed that PD-L1 and CD47 were increased in MRNA, total protein and membrane protein levels in TST knockout T24 cell (Figure8C,E,F), and TST was significantly negatively correlated with PD-L1 and CD47 (Figure 8I,J). In addition, CD47 increased in mRNA, total protein and membrane protein levels in S100A16 knockout T24 cells (Figure 8D,G), and S100A16 was positively correlated with CD47 (Figure 7K), while PD-L1 did not change (Figure 8D,H). To sum up, these findings indicate that TST and S100A16 play a key role in the regulation of tumor immune microenvironment of BCa.

\section{Discussion}

Metabolic dysregulation is emerging as a novel hallmark in tumor and plays important role in tumorigenesis and tumor progression (18). Previous studies had reported that the fatty acid metabolism was highly activated in BCa tissues compared with paracancerous tissues (11), and the alteration in 
glucose and pyruvate metabolism could promote the progression of BCa cells (19). Considering the vital role of the metabolic disorder in $\mathrm{BCa}$, it is important to identify metabolic-related prognostic biomarkers for the prognosis and treatment of BCa. In this study, we identified two clusters based on the RNA expression of 1257 MAGs. The clinicopathological features and prognoses were significantly different between the two clusters, revealing that the RNA expression of 1257 MAGs remarkedly related to features of BCa. GSVA demonstrated that clusters A and B were primarily enriched for infection-and metabolismrelated biological processes, respectively, which may partly explain the differences in clinical pathology and prognosis between the two clusters. However, according to their metabolic classification, tang et al. found that the biological processes of the three clusters were all significantly related to the metabolic pathways (13). The reason for this difference may be due to inconsistent algorithms for metabolic classification.

Then, we used the hub DEGs between two clusters to construct a metabolic-associated signature that had good performance in prognostic stratification in the combined data set and IMvigor210 trial, in which BCa patients with high-risk scores showed significantly poorer survival outcomes than patients with low-risk scores. Moreover, patients with high-risk scores were more prone to have advanced clinicopathological features and have SD or PD when receiving PD-L1 inhibitors, which revealed that patients with low-risk scores are more to respond to immunotherapy.. In addition, the IC50 values of Cisplatin and Gemcitabine were significantly lower in patients with high-risk scores, which revealed that patients with high-risk scores are more to respond to chemotherapy. Based on the above results, it may be helpful to guide the individualized treatment of $\mathrm{BCa}$. Tang et al. also predicted the treatment response of patients with $\mathrm{BCa}$ by metabolic classification (13). Their study found that patients with M1 subtype showed the best predictive response to immunotherapy and were the least sensitive to cisplatin. By contrast, patients with M2 subtypes had the worst predictive response to immunotherapy, but were more sensitive to chemotherapeutic agents such as cisplatin and doxorubicin. Similar conclusions were reached, although a different algorithm was used as in our study.

High needs of cancer cells for nutrients contribute to the metabolic reprogramming of both cancer cells and TIICs, which may shape the density/composition of TIICs, or directly modulate the functionality of TIICs to facilitate immunosuppression and tumor progression (20-22). TAMs and regulatory $T$ cells (Tregs) represent the main fraction of the TIICs in the tumor microenvironment to support tumor growth and metastasis through promoting immunosuppression $(23,24)$. It has been reported that the activation of fatty acid $\beta$-oxidation in TAMs and regulatory $T$ cells is important for their survival and immunosuppression function in tumor progression $(25,26)$. In our study, the composition of TIICs between clusters or risk groups was distinct, and patients in cluster A or high-risk group had significantly higher proportions of TAMs and regulatory T cells, which may contribute to the poor survival outcomes of these patients. In addition, low mutation count and high proportion of TAMs and regulatory T cells in high-risk group partly explained the low proportion of CR/PR and poor survival outcomes of high-risk patients treated with PD-L1 inhibitor. Similarly, Tang et al. found a positive correlation between high immune infiltration and poor therapeutic response of immune checkpoint inhibitors in the prediction of 
treatment response (13). Thus, the metabolic disorder could shape the composition of TIICs and the metabolic-associated signature may have the potential to facilitate personalized immunotherapy in BCa.

Experimental studies of hub genes in the metabolic-associated signature were performed to explore their immunological effects. TST is an abundant mitochondrial enzyme that catalyzes the transfer of sulfur in multiple biological pathways, especially the conversion of sulfurized Glutathione to thiosulfate and glutathione in a sulfite-dependent manner (27). Dysfunction of TST was highly associated with the development of metabolic diseases such as obesity and diabetes (28). It is reported that the TST may play a role in the development of colorectal cancer and glioma $(29,30)$. S100A16 belongs to the small acidic $S 100$ calcium-binding protein family that is involved in chromosomal instability and rearrangements, contributing to malignant transformation of cells $(31,32)$. Overexpression of S100A16 in diverse cancer cells reveals its close relationship with the tumorigenesis and tumor progression (33-36). However, the functions of these two genes in BCa cells have not been explored. In this study, we explored the biological functions and immunological effects of TST and S100A16 in BCa cells. The results demonstrated that TST knockdown promoted the invasion, migration and EMT of the BCa cell while S100A16 knockdown inhibited these processes. Studies have shown that TST can detoxify $\mathrm{H}_{2} \mathrm{~S}(29)$, and

$\mathrm{H}_{2} \mathrm{~S}$ is crucial for the occurrence and progression of $\mathrm{BCa}$, especially in inducing cell proliferation and promoting invasion (37-39). Similarly, some studies have found that S100A16 promotes drug resistance in NMIBC (40), and S100A16 also promotes the metastasis and progression of pancreatic cancer by inducing EMT (41). In addition, the results of western blot and flow cytometry showed that TST knockdown promoted and S100A16 knockdown inhibited the protein expressions of critical immune checkpoint genes that have functions in immunosuppression. Thus, dysexpression of TST or S100A16 may contribute to the tumor progression, immunosuppression and dysregulation of immune microenvironment in $\mathrm{BCa}$.

Despite the above important findings, our study has certain limitations. First, this is a retrospective study, which may lead to deviations due to incomplete information. Second, the sample size of the model needs to be further expanded before clinical application. Finally, additional functional studies and mechanism analysis are still needed to explore the potential molecular mechanisms of the immune effects of TST and S100A16, which are of great significance for the development of targeted therapy.

\section{Conclusion}

The metabolic-associated signature had the potential to help outcome prediction and personalized immunotherapy in $\mathrm{BCa}$. Metabolism disorder contributed to the dysregulation of immune microenvironment, thus leading to immunosuppression. The hub genes in the metabolic-associated signature could modulate the tumor immune microenvironment.

\section{Materials And Methods}

\section{Ethics}


This study was reviewed and approved by the Ethics Committee of Shanghai Tenth People's Hospital, and all patients signed written informed consent.

\section{Data Collection}

We collected 135 BCa samples confirmed histologically in Shanghai Tenth People's Hospital between November 2019 and April 2021 according to the following criteria: (1) histologically confirmed BCa; (2) samples were available for RNA-sequence; (3) availability of clinical and follow-up data. The processes of total RNA extraction, paired-end libraries generation and RNA-sequence can be found in our previous publications (16).

RNA-sequence data (FPKM standardized data) for BCa patients were downloaded from The Cancer Genome Atlas (TCGA) (http://www.cancergenome.nih.gov). The microarray data sets GSE48075 and GSE13507 were obtained from the GEO database (https://www.ncbi.nlm.nih.gov/). The transcriptomic data of IMvigor210 trial, which included metastatic urothelial cancer patients treated with atezolizumab (PD-L1 inhibitor), were downloaded from the http://research-pub.gene.com/IMvigor210CoreBiologies. The list of MAGs was extracted from the metabolic pathways Gene set enrichment analysis (GSEA, c2.cp.kegg.v7.1.symbols.gmt). We used the ComBat, a batch effect correcting tool in the sva R package v3.38, to adjust batch effects and combine these data sets.

\section{Unsupervised clustering}

We used the 'ConsensusClusterPlus' R package v.1.50.0 (Pearson correlation, 50 iterations and $80 \%$ resample rate) to divide BCa patients into different clusters based on the expression of MAGs. Gene Set Variation Analysis (GSVA) was performed to quantitatively investigate different biological pathways between clusters using 'GSVA' R package v.1.38.2. single-sample gene set enrichment analysis (ssGSEA) was used to evaluate the proportions of 28 TIICs in each BCa sample.

\section{Construction of the metabolic-associated signature}

The differentially expressed genes (DEGs, $\mid$ Log2FC|>1.5 and adjusted $\mathrm{P}<0.05)$ between clusters were identified using 'limma' R package v3.46. The random forest along with Boruta feature selection was performed using the 'Boruta' R package v7.0 to obtain the most important DEGs involved in discriminating clusters, followed by the univariate cox regression. In this way, the selected genes can be divided into two groups based on the hazard ratio (HR) of the univariate cox regression: protective gene $(H R<1)$ and risk gene $(H R>1)$. The metabolic-associated signature was developed using the following formula:

metabolic score $=$ scale ()

Where $X$ is the expression of each selected gene with $H R>1$, and $Y$ is the expression of each selected gene with $\mathrm{HR}<1$. This formula was used to calculate the risk score of each patient. Then, patients were divided 
into low- and high-risk groups according to the optimal cutoff value of risk score calculated by the 'survminer' R package v0.4.8.

\section{Chemotherapeutic Response Prediction}

The chemotherapy response of each sample was predicted based on the Genomics of Drug Sensitivity in Cancer which is the largest public pharmacogenomics database. Two commonly used chemicals Cisplatin and Gemcitabine were selected to evaluated the IC50 of each sample using the 'pRRophetic' R package.

\section{Gene mutation in Cancer Cell Line Encyclopedia (CCLE)}

The gene expression data and mutation data of 592 carcinoma cell lines were down from CCLE (https://portals.broadinstitute.org/ccle). The risk scores of each carcinoma cell line were calculated using the formula mentioned above. Then, carcinoma cell lines were divided into low- and high-risk groups based on the median value of risk scores. The mutation counts were compared between low- and highrisk groups.

\section{Hub genes screening}

We used three algorithms, including support vector machine-based recursive feature elimination (SVMRFE, 'e1071' R package v1.7) algorithm, MeanDecreaseGini-based random forest-feature selection (RFSFS, 'randomForest' R package v4.6) and MeanDecreaseAccuracy-based RFS-FS, to rank genes with respect to the risk group. The top ten genes of each algorithm were put into a Venn online software (http://bioinformatics.psb.ugent.be/webtools/Venn/) to identify the overlapping hub genes. The GSEA (http://www.broadinstitute.org/GSEA) was used to investigate the enriched gene signatures.

\section{Bioinformatics analysis}

The Assistant for Clinical Bioinformatics is an online tool (www.aclbi.com) that contains gene expression data and survival information of various cancers in the TCGA database, and all data are analyzed by $R$ v4.0.3. This data set can provide survival analysis, correlation analysis, prognostic nomogram and tumor tissue immune cell infiltration.

Kaplan-Meier Plotter is an online tool (www.kmplot.com) that contains gene expression data and survival information of various cancers for analyzing the prognostic value of TST and S100A16 in BCa.

According to the median mRNA expression of $\mathrm{BCa}$ samples, they were divided into high expression group and low expression group, and then analyzed by Kaplan-Meier survival curve.

TIMER is an online tool (http://timer.cistrome.org) that contains a comprehensive analysis of immune infiltration in different types of cancer, and is used to analyze the association between TST and S100A16 and immune infiltration in $\mathrm{BCa}$. The associations between genetic markers and various immune cells are also recorded. 
GEPIA is an online tool (http://gepia2.cancer-pku.cn) that can provide tumor/normal differential expression analysis, survival analysis, correlation analysis. BCa patients were used to analyze the association between genes based on their mRNA expression.

\section{Immunohistochemistry (IHC)}

The Human Protein Atlas (http://www.proteinatlas.org) online database can obtain IHC slides and information to check the expression levels of TST and S100A16 in normal bladder tissues and BCa specimens. The score of the positive staining ratio of tumor cells is defined as: 0 (none); $1(<25 \%) ; 2$ $(25 \%-75 \%) ; 4$ (>75\%). The staining intensity score is determined as: 0 (Negative); 1 (Weak); 2 (Moderate) and 3 (Strong). The immune response score is evaluated by multiplying the staining intensity and the positive cell ratio score.

\section{Cell lines and culture}

Human HEK293T, human BCa cell line T24 were obtained from the American Type Culture Collection (ATCC, Rockville, USA). Human HEK293T is maintained in DMEM medium (Gibco, USA); T24 cells are cultured in RPMI-1640 (Gibco, USA). All cell lines were cultured in a humidified incubator containing $5 \%$ $\mathrm{CO}_{2}$ at $37^{\circ} \mathrm{C}$. All cell culture media contain $10 \%$ fetal bovine serum (FBS; Gibco) and $1 \%$ penicillin/streptomycin (Hyclone; GE Healthcare Life Sciences, Logan, UT, USA).

\section{Generation of TST and S100A16 knockout cell lines}

TST and S100A16 gene knockout T24 cell lines were produced using lenti CRISPR technology (42). In short, the oligonucleotides encoding gRNA (TST sgRNA1: 5'-TCCGGAACTGGCTGAAGGA-3'; TST sgRNA2: 5'-CATCAGGACTGGCAAGCTG-3'; S100A16 sgRNA1: 5'-AGGCCTTACCGACAGCATG-3'; S100A16 sgRNA3: 5'-CAGGACACAGGGAACCGGA-3') was constructed into the lentiviral CRISPR-puro vector. Then, the plasmid was co-transfected into HEK293T cells with packaging vectors including pSPAX2 and pMD2G. After 48 hours, the culture supernatant was harvested to infect the T24 cell line. Puromycin $(1 \mu \mathrm{g} / \mathrm{ml})$ was used to select lentivirus-transduced cells for 3 days and verified by Western blot.

\section{Real-time quantitative PCR (RT-qPCR)}

According to the manufacturer's instructions, total RNA was extracted from the cells using TRIzol reagent (Invitrogen, USA), and then reverse transcription was performed using HiScript ${ }^{\circledR} Q$ Select RT SuperMix (Vazyme Biotechnology Company, China) to synthesize cDNA samples. RT-qPCR was performed using SYBR qPCR Master Mix (Vazyme Biotech, China), and quantified by CFX real-time PCR detection system (BIO-RAD, USA). The primers used in this study are as follows: The primers were provided in Supplementary Table 1.

\section{Western Blot}


The cells were collected and lysed in lysis buffer (150 mM NaCl, 0.5\% NP-40, 1 mM EDTA, 10\% glycerophosphate, $50 \mathrm{mM}$ Tris-Cl, $\mathrm{pH} 7.4$ and protease inhibitor cocktail). After 30 minutes, the cell lysate was separated by centrifugation at $12,000 \mathrm{rpm} 4^{\circ} \mathrm{C}$ for 15 minutes. The protein concentration was quantified by BCA protein analysis kit (Beyotime, Shanghai, China). Separated by SDS-polyacrylamide gel electrophoresis (SDS-PAGE), and analyzed by immunoblotting. The western blot was detected using an electrochemiluminescence (ECL) imaging system (Tanon, Shanghai, China). Anti-TST (ab166625), CD47 (ab218810), PD-L1 (ab136845), E-cadherin (ab1416), N-cadherin (ab12221), Vimentin (ab92547), Slug (ab85936) antibodies were obtained from Abcam; anti-Tubullin (2148S) antibody was obtained from Cell Signaling Technology; anti-S100A16 (11456-1-AP) antibody was obtained from Proteintech.

\section{Flow cytometry}

Cells were washed in PBS and stained for cell surface CD 47 or PD-L1 on ice for 30 min in PBS plus $2 \%$ FACS. After washing in PBS samples were analysed on a BD LSR Fortessa and analysed in FlowJo. APC anti-human CD274 Antibody Obtained from Biolegend, Clone 29E.2A3, Cat\# 329708; PE anti-human CD47 Antibody was obtained from Biolegend, Clone: CC2C6, Cat\# 323108.

\section{Cell migration assay}

According to the manufacturer's instructions, cell migration was analyzed using the Transwell system (Corning, NY, USA). Resuspend the T24 cell line in serum-free medium, and then add $200 \mu$ l of cell suspension (containing $5 \times 10^{4}$ cells) to the upper Transwell chamber. The lower chamber contains $600 \mu \mathrm{l}$ of medium with $10 \% \mathrm{FBS}$. After 16 hours of incubation at $37^{\circ} \mathrm{C}$, the cells that migrated on the lower surface of the chamber were fixed with $4 \%$ paraformaldehyde and stained with crystal violet.

\section{Matrigel droplet invasion assay}

$5 \times 10^{4} \mathrm{~T} 24$ cells were suspended in $10 \mu \mathrm{l}$ Matrigel, seeded in a 24-well plate in the form of droplets, and the droplets were imaged using a microscope (Olympus Corporation) on day 0 and day 4 , as described previously $(43,44)$. The added medium was changed once a day. The migration of cells extending to the outside of the droplet was measured by the Image J software.

\section{Statistical analysis}

Statistical analysis was conducted with SPSS 23.0 (IBM Corp.) and R statistical software v3.6.1 (https://www.r-project.org/). The factors between clusters or risk groups were compared using the Chisquare test, the t-test or the Mann-Whitney $U$ test, as appropriate. Survival analyses were performed using the Kaplan-Meier method and Cox proportional hazard models. All tests were 2-tailed, and P values $<0.05$ were considered statistically significant.

\section{Abbreviations}


BCa: bladder cancer; TIICs: tumor-infiltrating immune cells; GSEA: gene set enrichment analysis; TST:Thiosulfate transferase; S100A16: S100 calcium-binding protein A16; NMIBC: non-muscle-invasive bladder cancer; TURBT: transurethral resection of bladder tumor; BCG: Bacillus CalmetteGuérin; MAGs: metabolism associated genes; TAMs: tumor-associated macrophages; TCGA: The Cancer Genome Atlas; sSGSEA: single-sample gene set enrichment analysis; DEGs: differentially expressed genes; HR: hazard ratio; CCLE: Cancer Cell Line Encyclopedia; IHC: Immunohistochemistry; RT-qPCR: Real-time quantitative PCR; CR: complete response; PR: response; PD: progressive disease; SD: stable disease; MMP9: Matrix metalloproteinase 9; DSS: disease-specific survival; EMT: epithelial-to-mesenchymal transition; Tregs: regulatory $\mathrm{T}$ cells.

\section{Declarations}

\section{Author Contributions}

Z.Z. and G.Y. collected and analyzed research data. L.S. was responsible for the interpretation of the results. G.Y. and Z.Z. drafted the manuscript. M.S, Y.F, W.R, W.H, L.L, L.J, Z.W, Y.X. and L.S. performed the study design and revised the manuscript.

\section{Funding}

This work was supported by funding from the Shanghai Science Committee Foundation (\#19411967700), Outstanding Talent of Shanghai Tenth People's Hospital (\#20215YPDRC048), Shanghai Youth Science and Technology Talents Sailing Program (\#20YF1437200).

\section{Availability of data and materials}

The data used to support the findings of this study are included in the article.

\section{Acknowledgements}

Not applicable

\section{Ethics approval and consent to participate}

This study was reviewed and approved by the Ethics Committee of Shanghai Tenth People's Hospital, and all patients signed written informed consent.

\section{Consent for publication}

All authors have seen the manuscript and approved to submit to your journal.

\section{Competing interests}

The authors declare that they have no competing interests. 


\section{Author details}

1. Department of Urology, Shanghai Tenth People's Hospital, School of Medicine, Tongji University, Shanghai, China

2. Institute of Urinary Oncology, School of Medicine, Tongji University, Shanghai, China

\section{References}

1. Siegel RL, Miller KD, Jemal A. (2020) Cancer statistics, 2020. CA Cancer J Clin 70: 7-30.

2. Cumberbatch MGK, et al. (2018) Epidemiology of Bladder Cancer: A Systematic Review and Contemporary Update of Risk Factors in 2018. Eur Urol 74: 784-795.

3. Babjuk M, et al. (2019) European Association of Urology Guidelines on Non-muscle-invasive Bladder Cancer (TaT1 and Carcinoma In Situ) - 2019 Update. Eur Uro/ 76: 639-657.

4. Witjes JA, et al. (2021) European Association of Urology Guidelines on Muscle-invasive and Metastatic Bladder Cancer: Summary of the 2020 Guidelines. Eur Urol 79: 82-104.

5. Guo Y, et al. (2019) Evaluation of Survival Outcomes With Trimodal Therapy as Primary Therapy for Non-organ-confined Bladder Cancer. Frontiers in oncology 9: 1315.

6. van Rhijn BW, et al. (2009) Recurrence and progression of disease in non-muscle-invasive bladder cancer: from epidemiology to treatment strategy. Eur Uro/ 56: 430-442.

7. Sanli O, et al. (2017) Bladder cancer. Nature reviews. Disease primers 3: 17022.

8. Jordan B, Meeks JJ. (2019) T1 bladder cancer: current considerations for diagnosis and management. Nature reviews. Urology 16: 23-34.

9. Sukjoi W, Ngamkham J, Attwood PV, Jitrapakdee S. (2021) Targeting Cancer Metabolism and Current Anti-Cancer Drugs. Advances in experimental medicine and biology 1286: 15-48.

10. Galon J, Bruni D. (2020) Tumor Immunology and Tumor Evolution: Intertwined Histories. Immunity 52: 55-81.

11. Cheng S, et al. (2019) Fatty acid oxidation inhibitor etomoxir suppresses tumor progression and induces cell cycle arrest via PPARy-mediated pathway in bladder cancer. Clinical science (London, England : 1979) 133: 1745-1758.

12. Massari F, et al. (2016) Metabolic phenotype of bladder cancer. Cancer Treat Rev 45: 46-57.

13. Tang C, Yu M, Ma J, Zhu Y. (2021) Metabolic classification of bladder cancer based on multi-omics integrated analysis to predict patient prognosis and treatment response. Journal of translational medicine 19: 205.

14. Leone R, Powell J. (2020) Metabolism of immune cells in cancer. Nature reviews. Cancer 20: 516531.

15. Netea-Maier RT, Smit JWA, Netea MG. (2018) Metabolic changes in tumor cells and tumor-associated macrophages: A mutual relationship. Cancer letters 413: 102-109. 
16. Zheng Z, et al. (2020) Dysregulation of the Immune Microenvironment Contributes to Malignant Progression and Has Prognostic Value in Bladder Cancer. Front Oncol 10: 542492.

17. Kessenbrock K, Plaks V, Werb Z. (2010) Matrix metalloproteinases: regulators of the tumor microenvironment. Cell 141: 52-67.

18. Faubert B, Solmonson A, DeBerardinis RJ. (2020) Metabolic reprogramming and cancer progression. Science 368.

19. Conde VR, et al. (2015) The progression from a lower to a higher invasive stage of bladder cancer is associated with severe alterations in glucose and pyruvate metabolism. Experimental cell research 335: 91-98.

20. Reina-Campos M, Moscat J, Diaz-Meco M. (2017) Metabolism shapes the tumor microenvironment. Current opinion in cell biology 48: 47-53.

21. Lyssiotis CA, Kimmelman AC. (2017) Metabolic Interactions in the Tumor Microenvironment. Trends in cell biology 27: 863-875.

22. Ma K, Zhang L. (2021) Overview: Lipid Metabolism in the Tumor Microenvironment. Advances in experimental medicine and biology 1316: 41-47.

23. Qian BZ, Pollard JW. (2010) Macrophage diversity enhances tumor progression and metastasis. Cell 141: 39-51.

24. Kitamura T, Qian BZ, Pollard JW. (2015) Immune cell promotion of metastasis. Nature reviews. Immunology 15: 73-86.

25. Michalek RD, et al. (2011) Cutting edge: distinct glycolytic and lipid oxidative metabolic programs are essential for effector and regulatory CD4+ T cell subsets. J Immuno/ 186: 3299-3303.

26. Huang SC, et al. (2014) Cell-intrinsic lysosomal lipolysis is essential for alternative activation of macrophages. Nature immunology 15: 846-855.

27. Libiad M, Sriraman A, Banerjee R. (2015) Polymorphic Variants of Human Rhodanese Exhibit Differences in Thermal Stability and Sulfur Transfer Kinetics. The Journal of biological chemistry 290: 23579-23588.

28. Kruithof PD, et al. (2020) Unraveling the role of thiosulfate sulfurtransferase in metabolic diseases. Biochimica et biophysica acta. Molecular basis of disease 1866: 165716.

29. Ramasamy S, Singh S, Taniere P, Langman M, Eggo M. (2006) Sulfide-detoxifying enzymes in the human colon are decreased in cancer and upregulated in differentiation. American journal of physiology. Gastrointestinal and liver physiology 291: G288-296.

30. Wróbel M, et al. (2014) Is development of high-grade gliomas sulfur-dependent? Molecules (Basel, Switzerland) 19: 21350-21362.

31. Katono K, et al. (2017) S100A16, a promising candidate as a prognostic marker for platinum-based adjuvant chemotherapy in resected lung adenocarcinoma. Onco Targets Ther 10: 5273-5279.

32. Chen H, Xu C, Jin Q, Liu Z. (2014) S100 protein family in human cancer. American journal of cancer research 4: 89-115. 
33. Qin F, et al. (2010) S100A8/A9 induces apoptosis and inhibits metastasis of CasKi human cervical cancer cells. Pathol Oncol Res 16: 353-360.

34. Saito K, et al. (2015) S100A16 is a Prognostic Marker for Lung Adenocarcinomas. Asian Pac J Cancer Prev 16: 7039-7044.

35. Sapkota D, et al. (2015) S100A16 promotes differentiation and contributes to a less aggressive tumor phenotype in oral squamous cell carcinoma. BMC cancer 15: 631.

36. Zhang H, Yang Y, Ma X, Xin W, Fan X. (2020) S100A16 Regulates HeLa Cell through the Phosphatidylinositol 3 Kinase (PI3K)/AKT Signaling Pathway. Med Sci Monit 26: e919757.

37. Liu H, Chang J, Zhao Z, Li Y, Hou J. (2017) Effects of exogenous hydrogen sulfide on the proliferation and invasion of human Bladder cancer cells. Journal of cancer research and therapeutics 13: 829832.

38. Gai J, et al. (2016) Expression profile of hydrogen sulfide and its synthases correlates with tumor stage and grade in urothelial cell carcinoma of bladder. Urologic oncology 34: 166.e115-120.

39. Wahafu W, et al. (2018) Increased HS and its synthases in urothelial cell carcinoma of the bladder, and enhanced cisplatin-induced apoptosis following HS inhibition in EJ cells. Oncology letters 15: 8484-8490.

40. Wang C, et al. (2019) S100A16 regulated by Snail promotes the chemoresistance of nonmuscle invasive bladder cancer through the AKT/Bcl-2 pathway. Cancer management and research 11: 2449-2456.

41. Li T, et al. (2021) S100A16 induces epithelial-mesenchymal transition in human PDAC cells and is a new therapeutic target for pancreatic cancer treatment that synergizes with gemcitabine. Biochemical pharmacology 189: 114396.

42. Shalem 0, et al. (2014) Genome-scale CRISPR-Cas9 knockout screening in human cells. Science 343: 84-87.

43. Rice MA, et al. (2019) Loss of Notch1 Activity Inhibits Prostate Cancer Growth and Metastasis and Sensitizes Prostate Cancer Cells to Antiandrogen Therapies. Molecular cancer therapeutics 18: 1230 1242.

44. Hsu EC, et al. (2020) Trop2 is a driver of metastatic prostate cancer with neuroendocrine phenotype via PARP1. Proceedings of the National Academy of Sciences of the United States of America 117: 2032-2042.

\section{Figures}




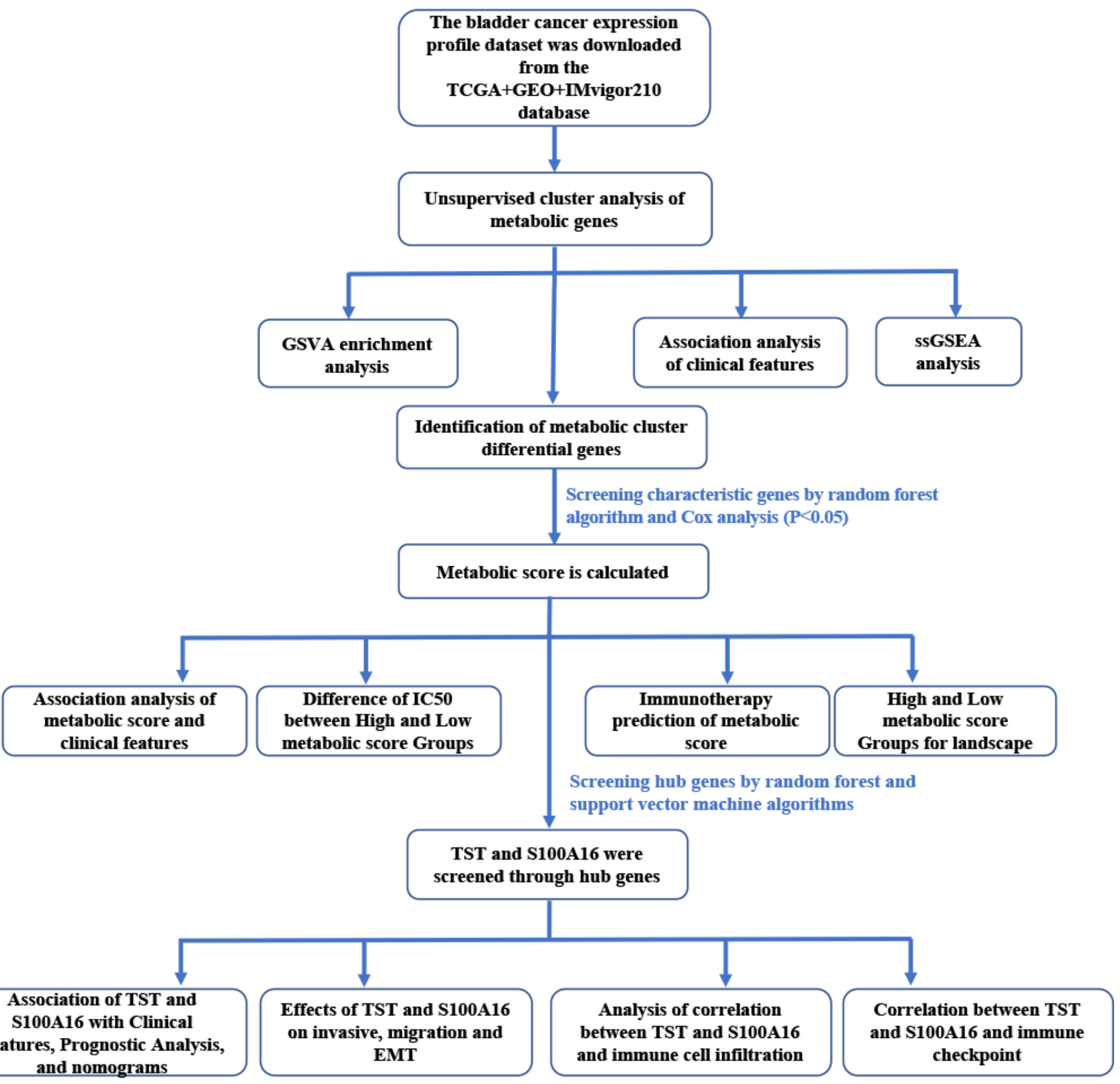

\section{Figure 1}

Workflow schematic 


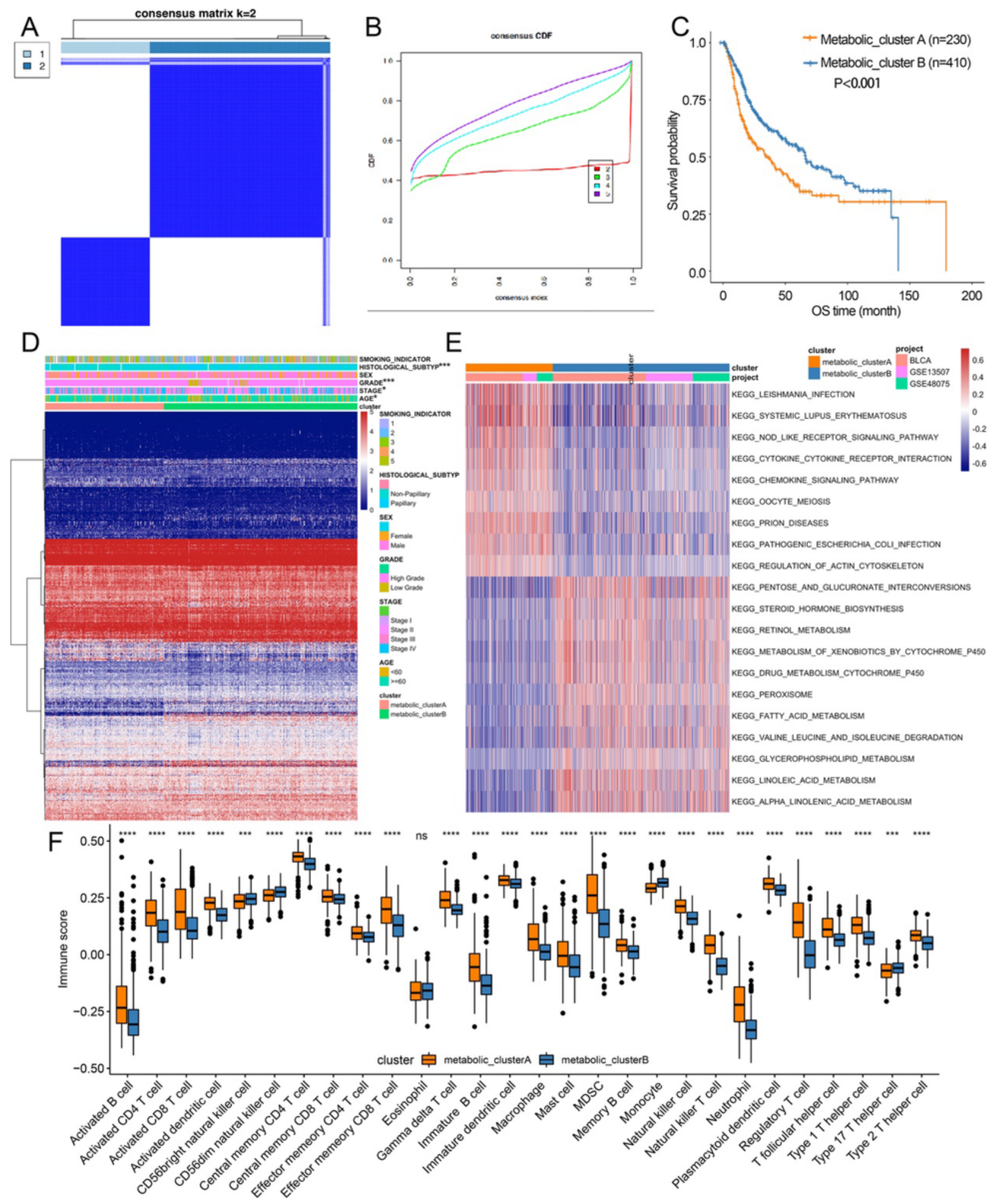

Figure 2

Differential clinicopathological features, biological pathways, immune microenvironment and survival outcomes of bladder cancer patients in the cluster A/B subgroups. (A) Consensus clustering matrix for $k=2$. (B) Cumulative distribution function of the consensus index. (C) Kaplan-Meier overall survival curves of the two clusters. (D) Clinicopathological features of the two clusters. (E) Enriched biological pathways of the two clusters. (F) Tumor-infiltrating immune cells of the two clusters. ${ }^{*} \mathrm{P}<0.05,{ }^{*} *{ }^{*} \mathrm{P}<0.001$. 

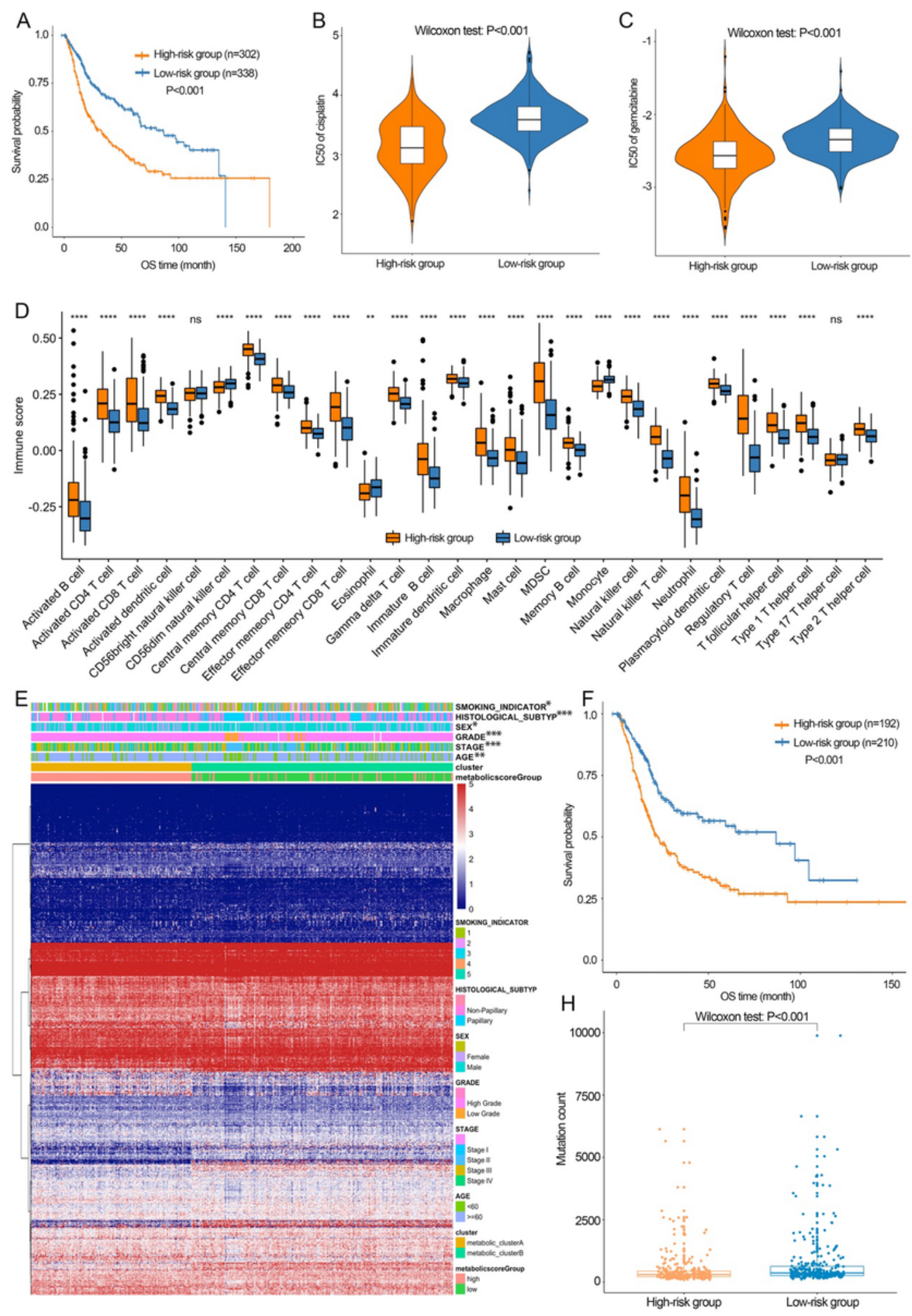

\section{Figure 3}

Performance of the metabolic-associated signature. (A) Kaplan-Meier overall survival curves of the two risk groups. (B) Cisplatin IC50 of the two risk groups. (C) Gemcitabine IC50 of the two risk groups. (D) Tumor-infiltrating immune cells of the two risk groups. (E) Clinicopathological features of the two risk groups in TCGA. (F) Kaplan-Meier overall survival curves of the two risk groups in TCGA. (G) Mutation 
count of the two risk groups in Cancer Cell Line Encyclopedia. TCGA: The Cancer Genome Atlas; *P<0.05, $\star * P<0.01, * * * P<0.001$.
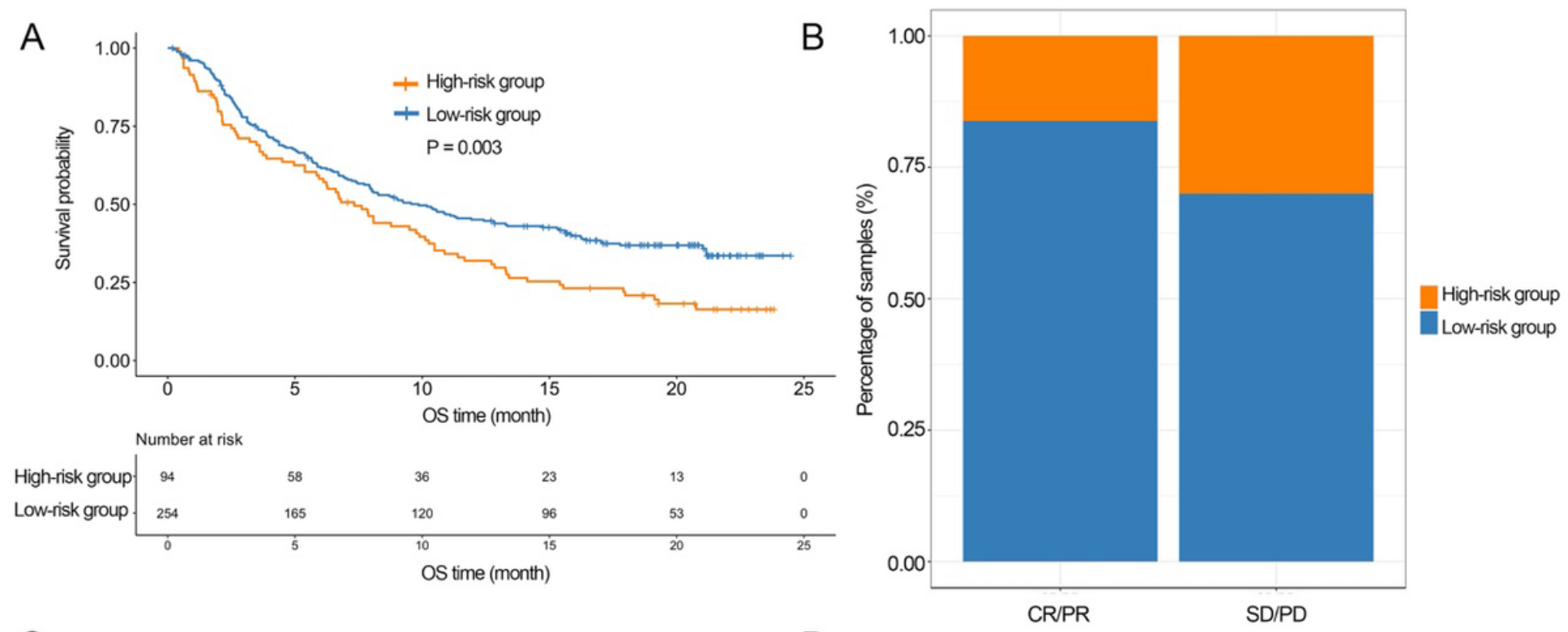

C
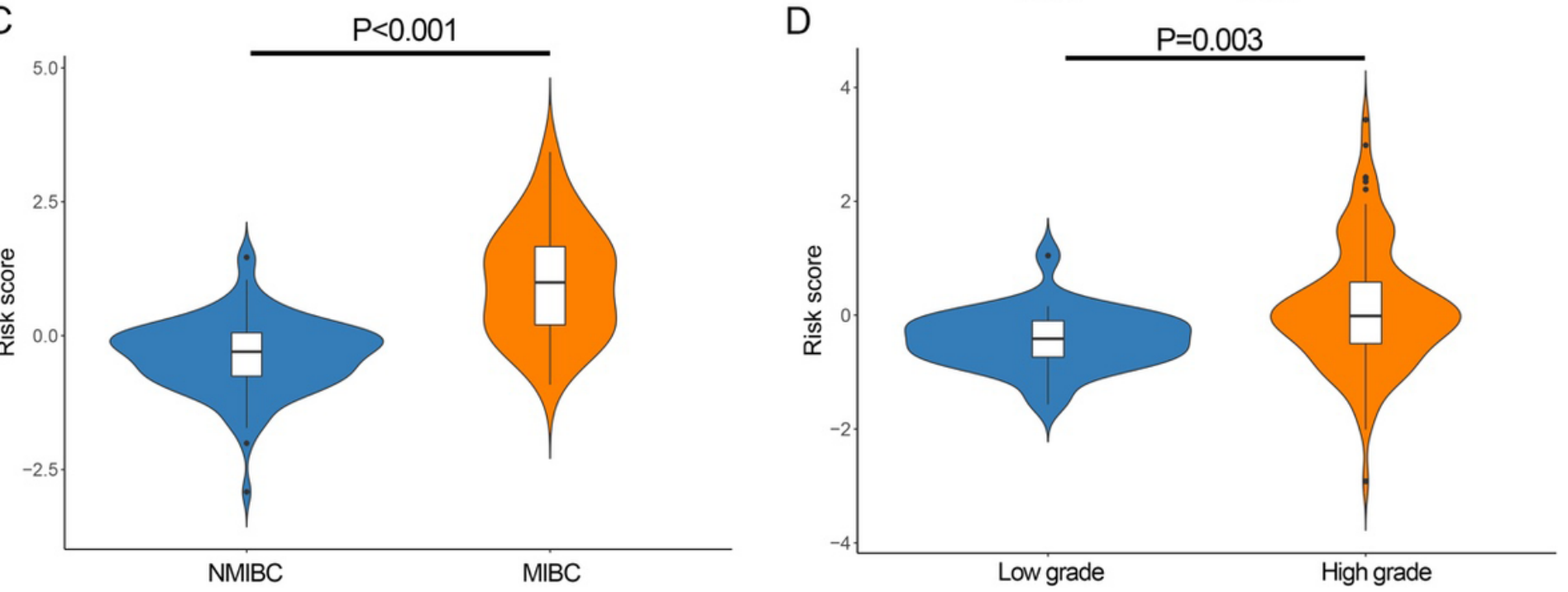

\section{Figure 4}

Validation of the metabolic-associated signature. (A) Kaplan-Meier overall survival curves of the two risk groups in IMvigor210 trial. (B) Response to PD-L1 inhibitor of the two risk groups in IMvigor210 trial. (C) Risk scores of the NMIBC and MIBC patients in our center. (D) Risk scores of the low-and high-grade bladder cancer patients in our center. NMIBC: non-muscle-invasive bladder cancer; MIBC: muscle-invasive bladder cancer. 

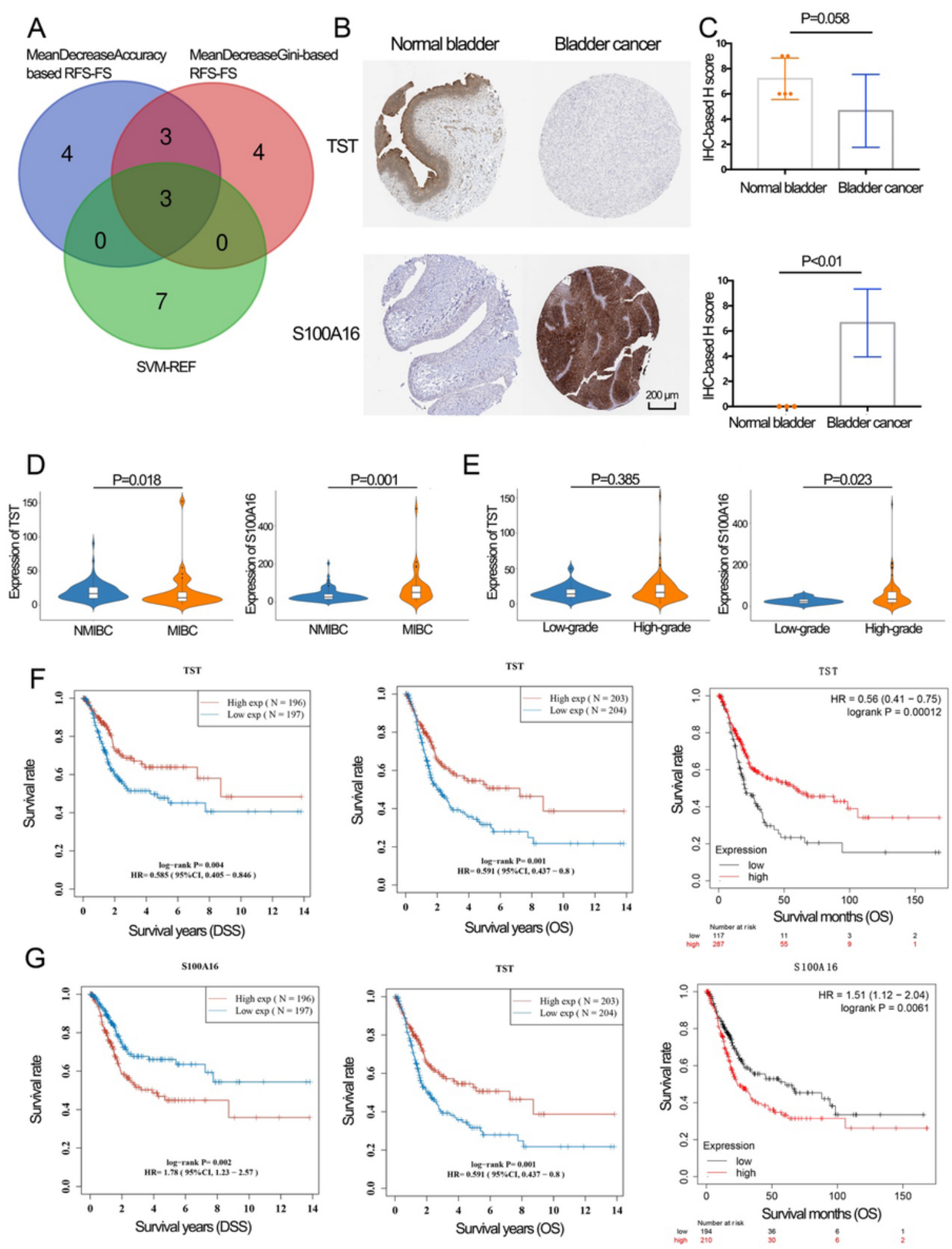

\section{Figure 5}

Validation of hub genes in metabolic-associated signature. (A) TST, S100A16 and MMP9 were obtained by hub genes screening. (B) IHC of TST and S100A16 in human protein atlas. (C) IHC-based H score of TST and S100A16. (D,E) The expression of TST and S100A16 in different T stages (D) and Grade (E). (F, G) OS and DSS curves in TST (F) and S100A16 (G) high and low expression bladder cancer cases from the Assistant for Clinical Bioinformatics (www.aclbi.com) and Kaplan-Meier plotter database 
(http://kmplot.com/analysis/). OS: Overall survival; DSS: disease-specific survival; Error bar \pm SD; ${ }^{*} \mathrm{P}<0.05$.

A

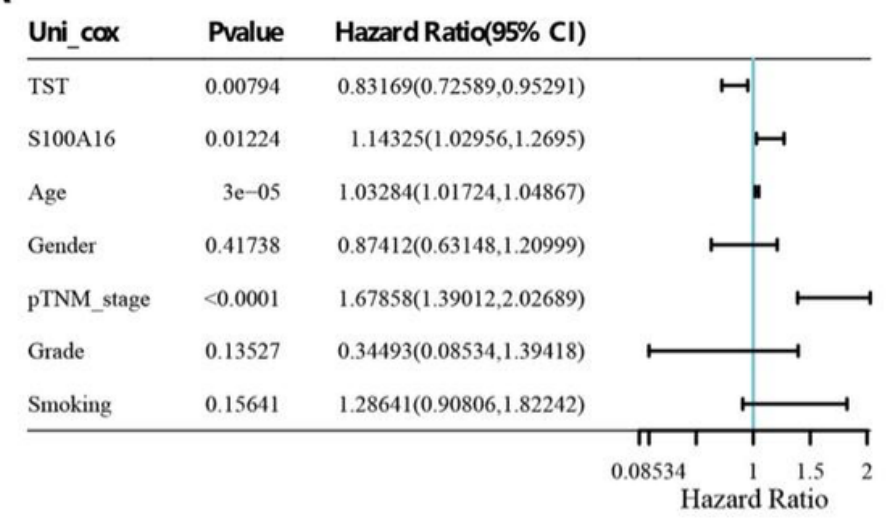

C

Points

TST

S100A16

Age

Total Points

Linear Predictor

1-year survival Pro

3-year survival Pro

5-year survival Pro
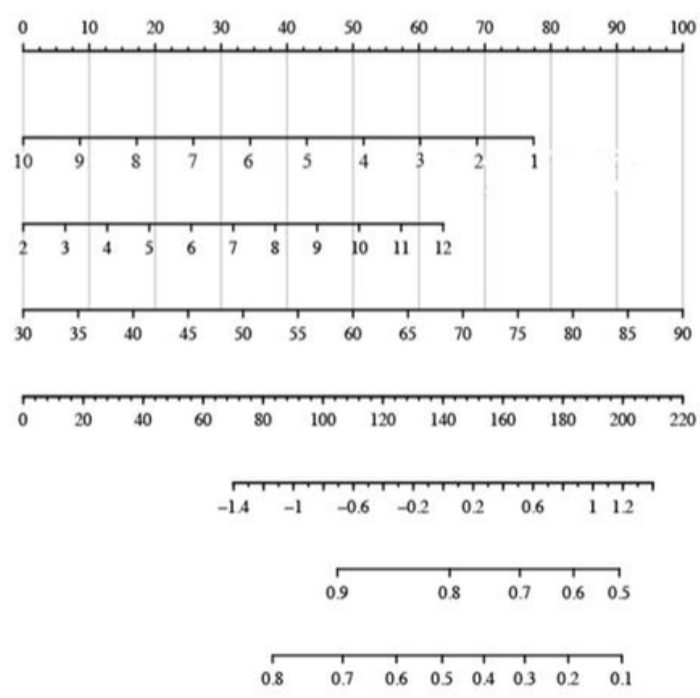

\begin{tabular}{lllllllll}
\hline 0.8 & 0.7 & 0.6 & 0.5 & 0.4 & 0.3 & 0.2 & 0.1
\end{tabular}
B

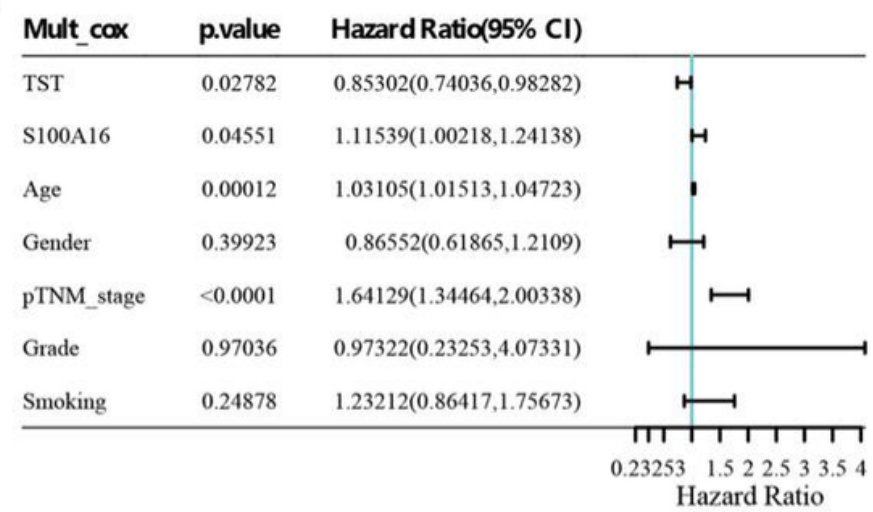

D

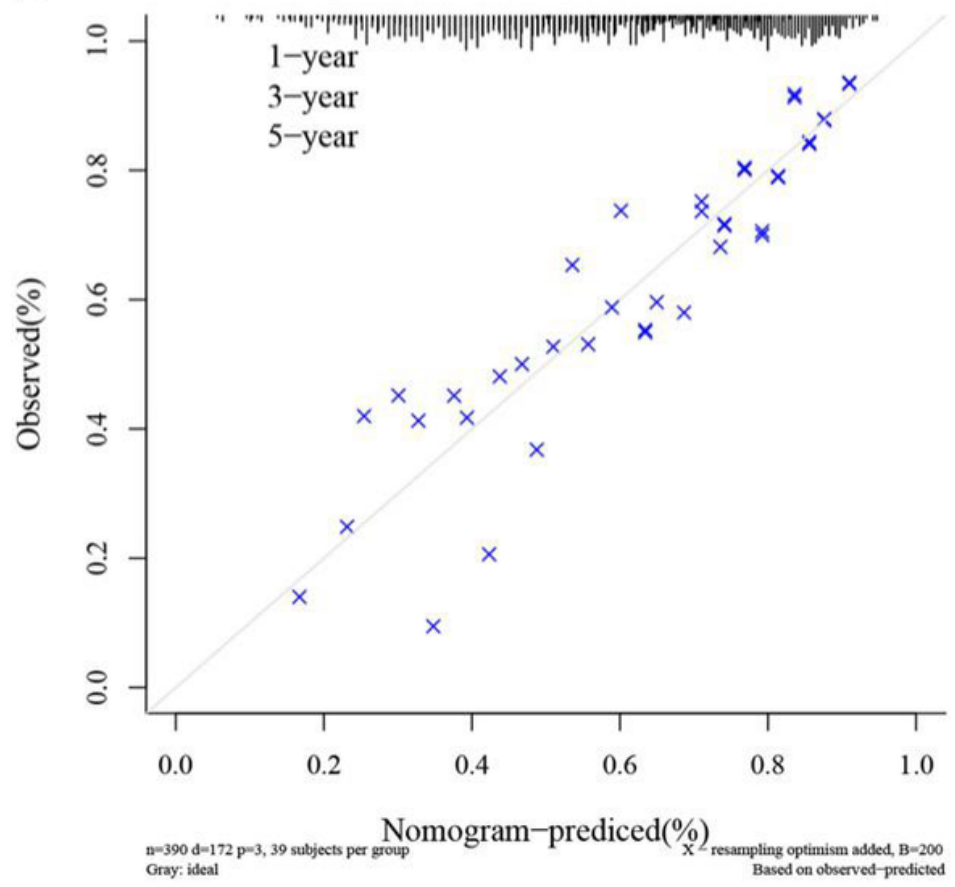

\section{Figure 6}

Construction of prognostic nomogram for BCa in TCGA. $(A, B)$ The outcomes of univariate and multivariate Cox proportional hazards regression. (C) Establish nomogram for $\mathrm{BCa}$ patients based on age, TST and S100A16. (D) Nomogram can better predict the 1-year, 3-year and 5-year average survival time of BCa patients. The HRs are presented as the means ( $95 \%$ confidence interval). 
A

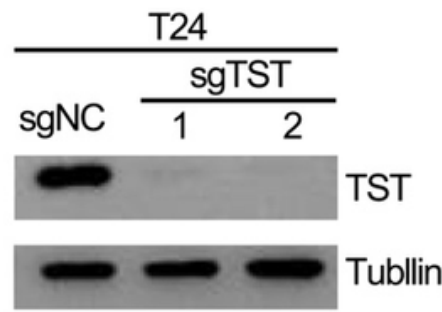

T24
sgNC $\frac{\mathrm{sgS} 100 \mathrm{~A} 16}{1} 2$

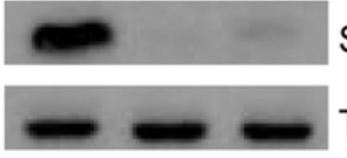

S100A 16

Tubllin
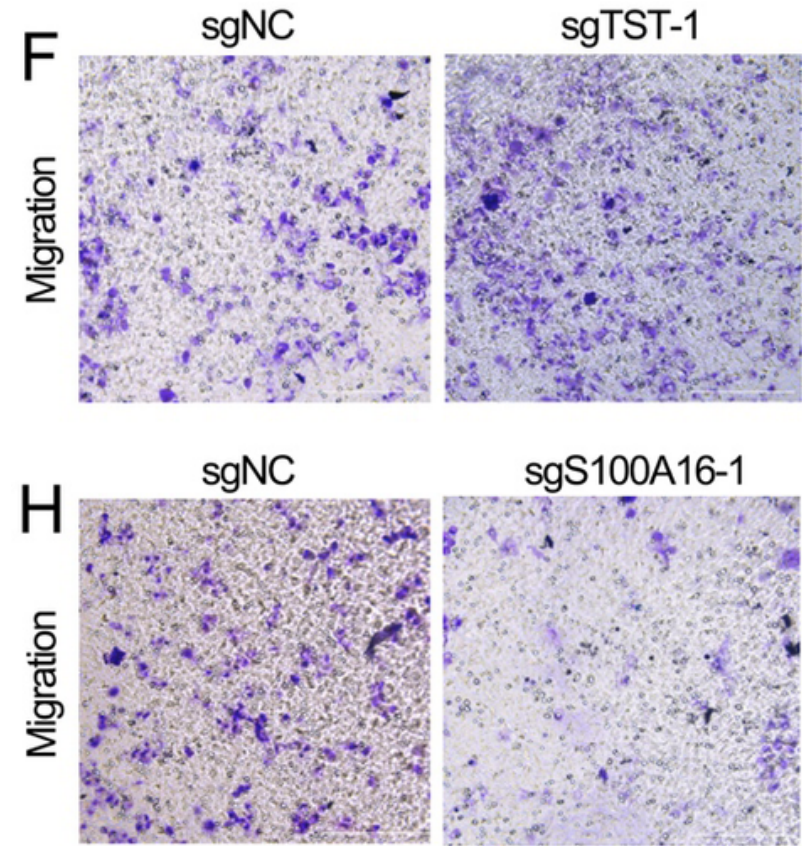

sgS100A16-1

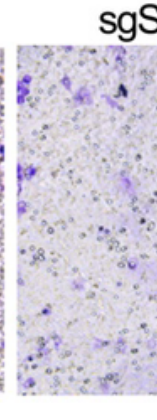

$\mathrm{J}$
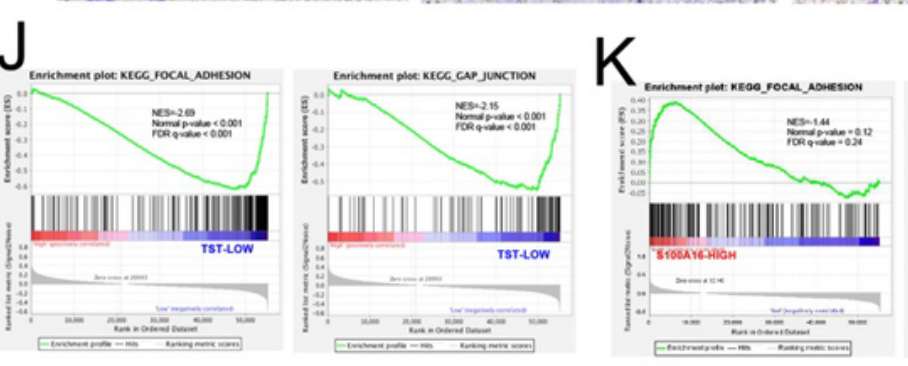
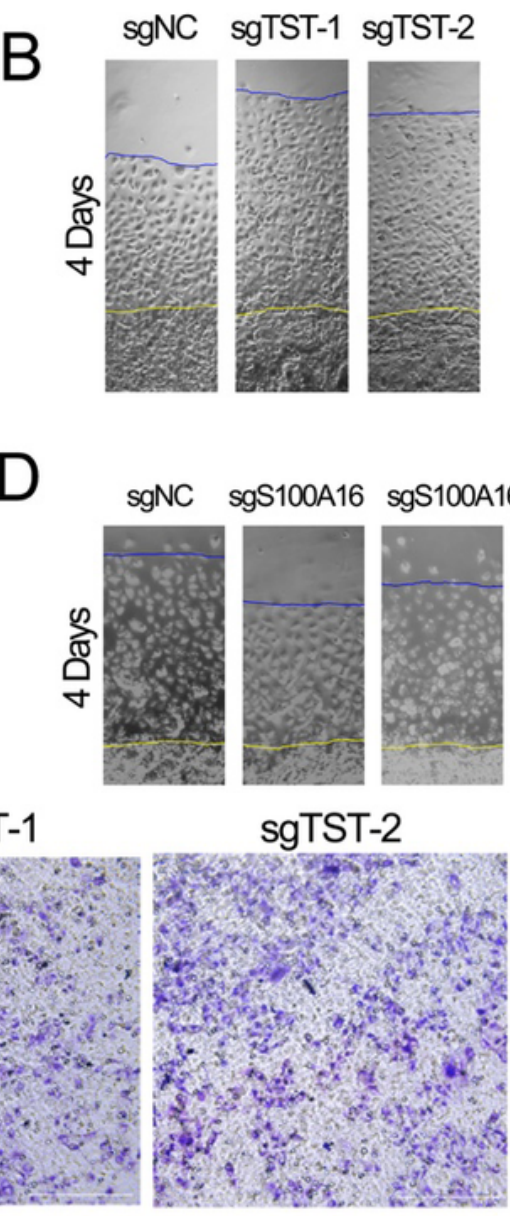

D

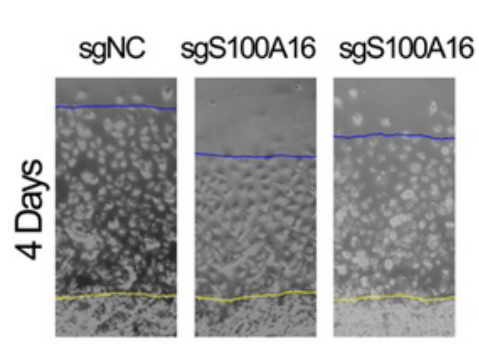

sgTST-2

sgS100A16-2
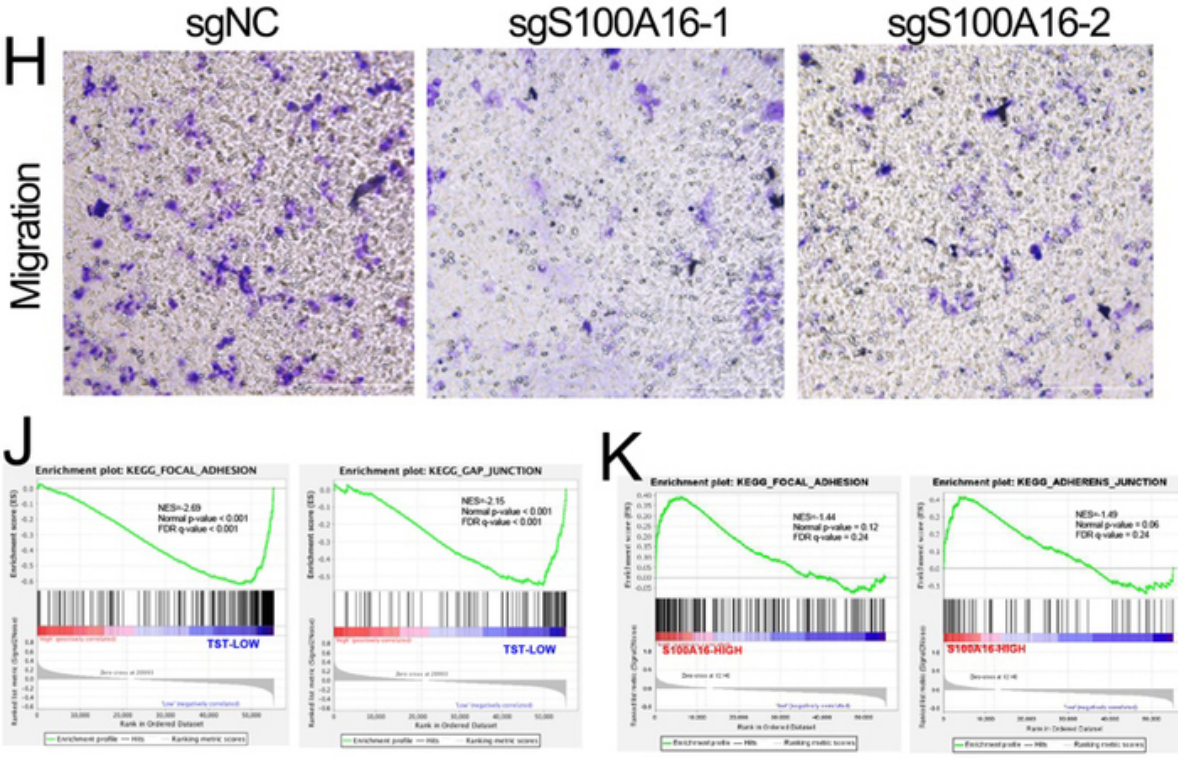
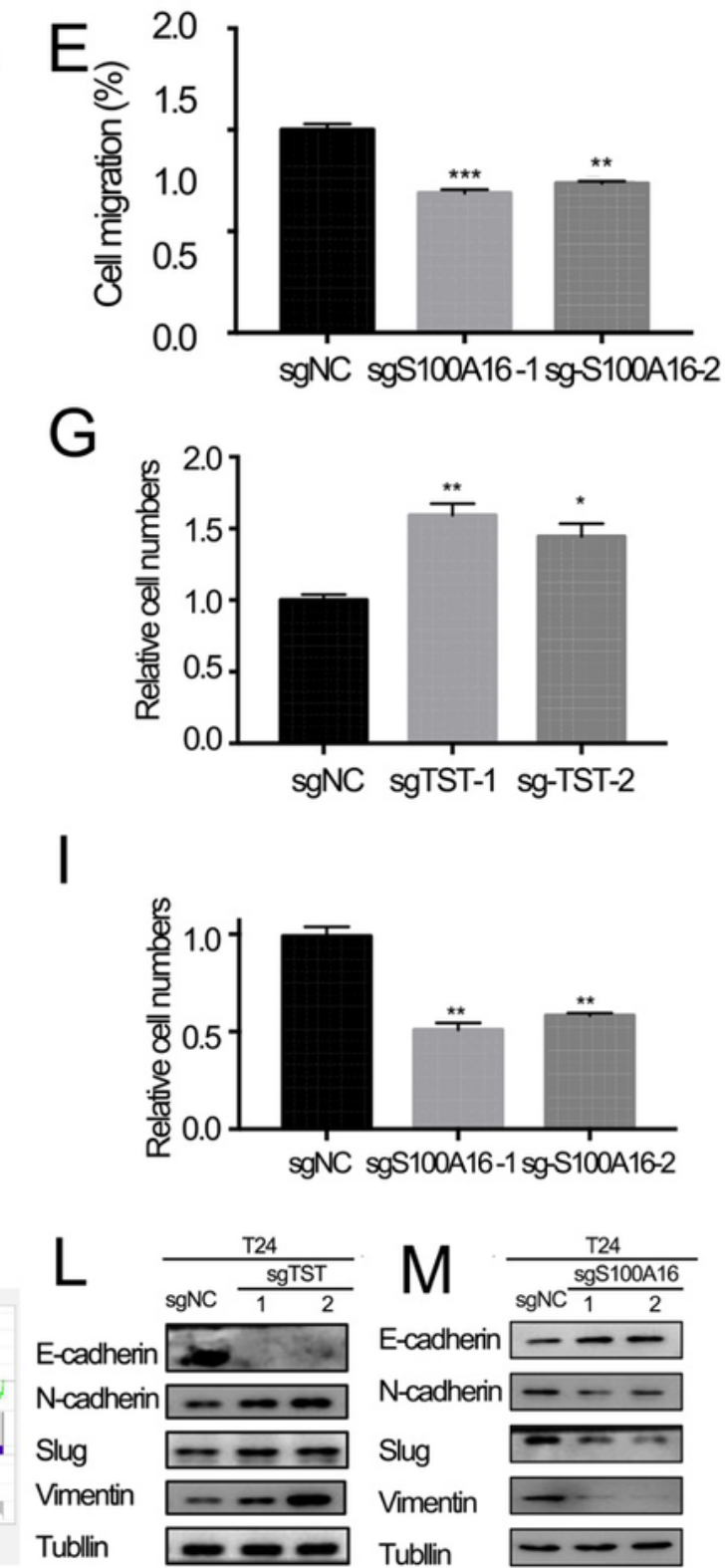

G

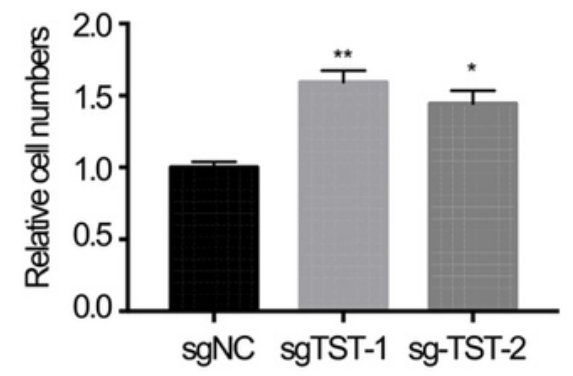

\section{Figure 7}

Downregulation of TST attenuate the invasion, migration and EMT of BCa cells, while S100A16 inhibited those processes. (A) Western blot analysis confirmed the efficiency of knocking out TST and S100A16 in T24 cells. (B-E) The invasion ability of T24 cells knocked out TST (B,C) and S100A16 (D,E) was detected by Matrigel droplet invasion. (F-I) The migration ability of T24 cells knocked out TST (F,G) and S100A16 $(\mathrm{H}, \mathrm{I})$ was detected by Transwell. $(\mathrm{J}, \mathrm{K})$ GSEA showed that TST $(\mathrm{J})$ and S100A16 (K) were correlated with 
FOCAL ADHESION, GAP JUNCTION and ADHESION JUNCTION of bladder cancer. (L,M) The expression of EMT-related marker protein was detected in T24 cells with TST (L) and S100A16 (M) knocked out. Data are given in mean standard deviation. ${ }^{*} \mathrm{P}<0.05 \rrbracket^{\star * *} \mathrm{P}<0.01{ }^{\star} * * * \mathrm{P}<0.001$.

A

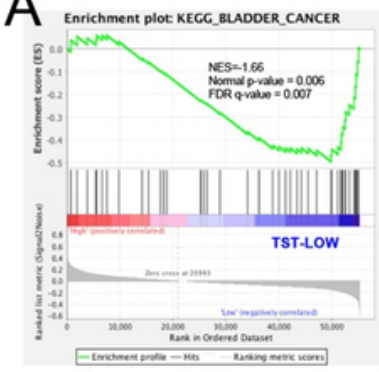

C
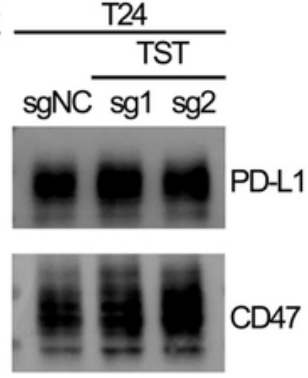

$\longrightarrow-$ Tubllin
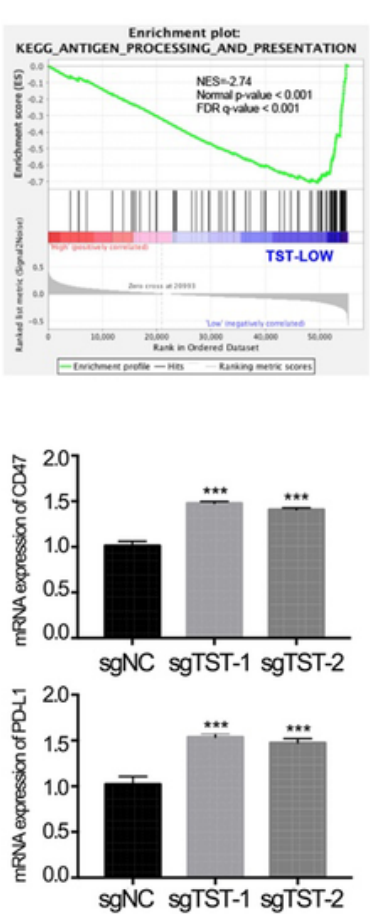

B
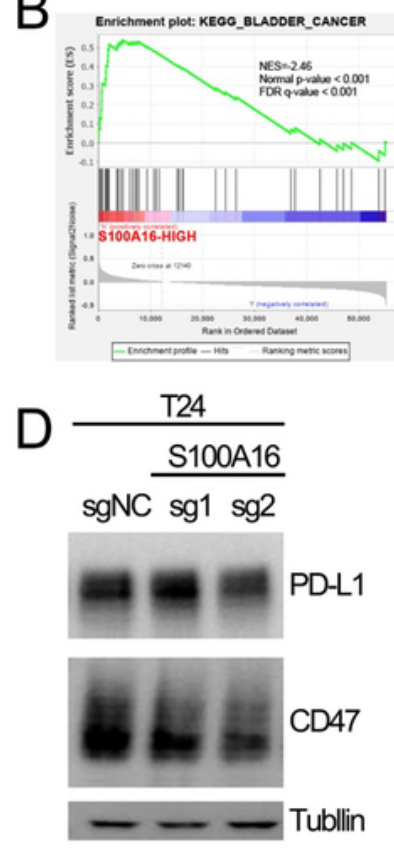
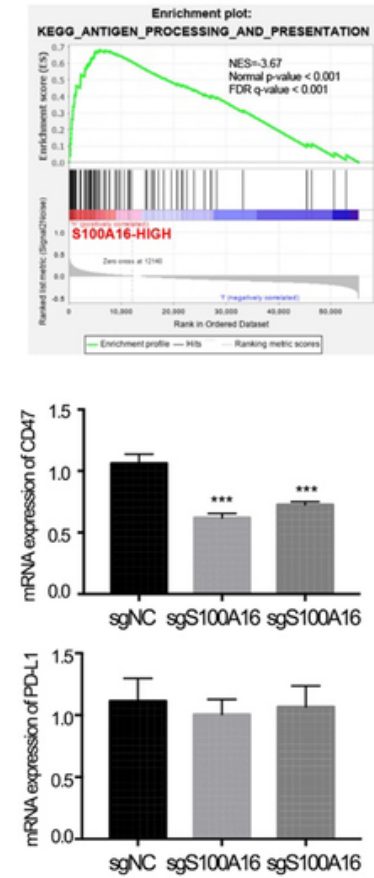
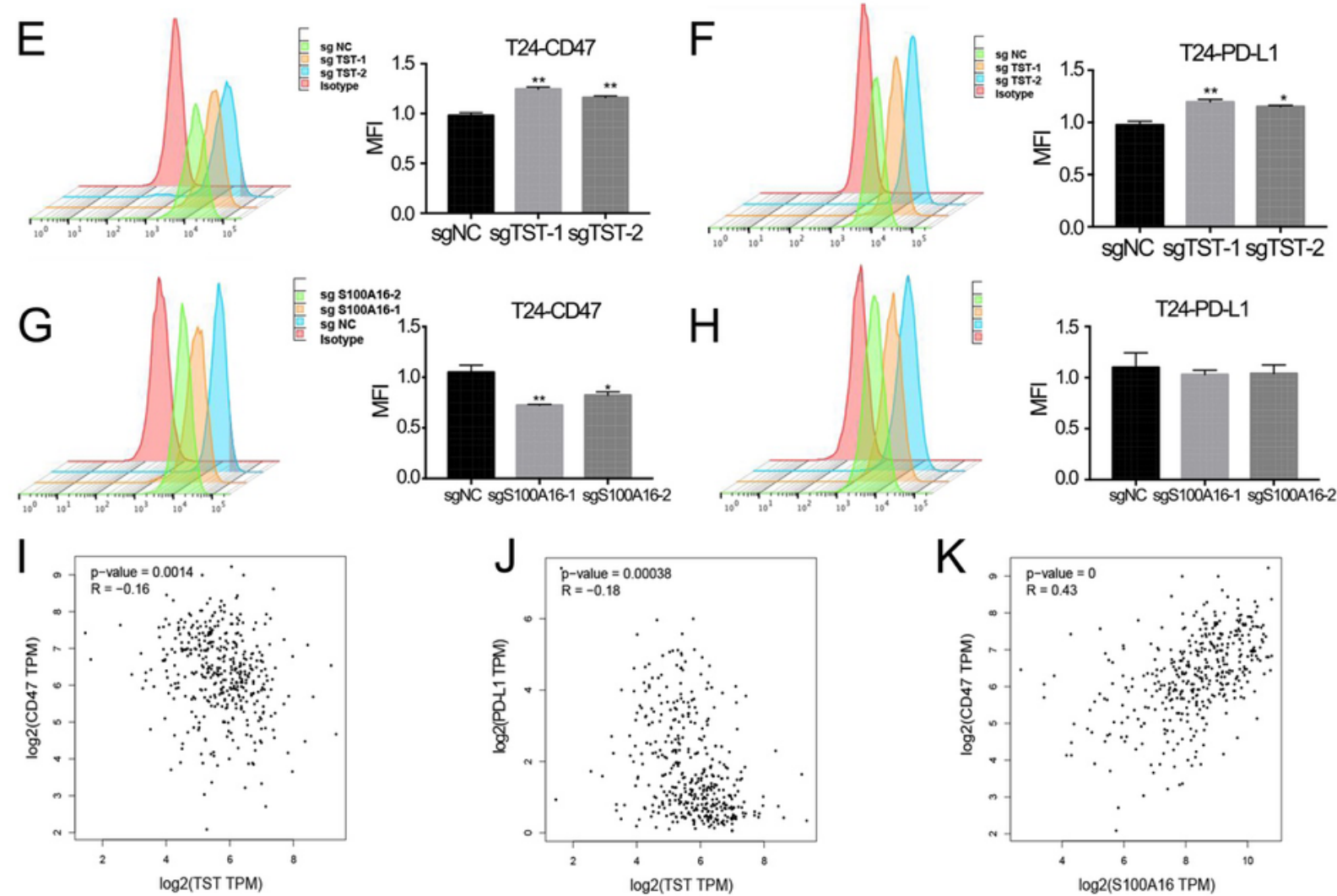

Figure 8

Correlation between TST and S100A16 and tumor immune microenvironment of BCa. $(A, B)$ GSEA analysis of TST (A) and S100A16 (B). (C,D) The total protein and mRNA expression levels of PD-L1 and 
CD47 were detected in TST (C) and S100A16 (D) knocked out T24 cells, respectively. (E-H) The membrane protein expression level of PD-L1 and CD47 were detected in TST $(E, F)$ and S100A16 $(G, H)$ knocked out T24 cells, respectively. (I-K) TCGA database showed that TST was negatively correlated with PDL1 (I) and CD47 (J), and S100A16 was positively correlated with CD47 (K).

\section{Supplementary Files}

This is a list of supplementary files associated with this preprint. Click to download.

- Supplementarymaterials.docx 